\title{
Effect of Facet Displacement on Radiation Field and Its Application for Panel Adjustment of Large Reflector Antenna
}

\author{
Wei WANG ${ }^{1,2} \cdot$ Peiyuan LIAN $^{1} \cdot$ Shuxin ZHANG $^{1,2} \cdot$ Binbin XIANG $^{1,3} \cdot$ \\ Qian $\mathbf{X U}^{3}$
}

Received: 9 May 2016/Revised: 27 February 2017/ Accepted: 2 April 2017/Published online: 13 April 2017

(c) The Author(s) 2017. This article is an open access publication

\begin{abstract}
Large reflector antennas are widely used in radars, satellite communication, radio astronomy, and so on. The rapid developments in these fields have created demands for development of better performance and higher surface accuracy. However, low accuracy and low efficiency are the common disadvantages for traditional panel alignment and adjustment. In order to improve the surface accuracy of large reflector antenna, a new method is presented to determinate panel adjustment values from far field pattern. Based on the method of Physical Optics (PO), the effect of panel facet displacement on radiation field value is derived. Then the linear system is constructed between panel adjustment vector and far field pattern. Using the method of Singular Value Decomposition (SVD), the adjustment value for all panel adjustors are obtained by solving the linear equations. An experiment is conducted on a $3.7 \mathrm{~m}$ reflector antenna with 12 segmented panels. The results of simulation and test are similar, which shows that the presented method is feasible. Moreover, the
\end{abstract}

Supported by National Natural Science Foundation of China (Grant Nos. 51490661, 51490660, 51205301), National Key Basic Research Program of China (973 Program, Grant No. 2015CB857100), and Special Funding for Key Laboratory of Xinjiang Uygur Autonomous Region, China (Grant No. 2014KL012).

Shuxin ZHANG

sxzhang@xidian.edu.cn

1 Key Laboratory of Electronic Equipment Structure Design of Ministry of Education, Xidian University, Xi'an 710071, China

2 Collaborative Innovation Center of Information Sensing and Understanding, Xidian University, Xi' an 710071, China

3 Xinjiang Astronomical Observation, Chinese Academy of Sciences, Urumqi 830011, China discussion about validation shows that the method can be used for many cases of reflector shape. The proposed research provides the instruction to adjust surface panels efficiently and accurately.

Keywords Reflector antennas - Surface accuracy · Radiation field - Reflector antenna mechanical factors . Electromechanical effects $\cdot$ Panel adjustment $\cdot$ Singular value decomposition (SVD)

\section{Introduction}

With the development of such fields as deep space communication, remote sensing and radio astronomy etc., many large reflector antennas take a great role because they can provide the enhanced data transmission, very high gain and lower noise radiations [1, 2]. However, there are many mechanical factors which affect strongly the electrical performance of reflector antennas [3-6]. In order to meet the requirements of science and technology, reflector antennas are correspondently designed larger and larger in size. It is very difficult for traditional techniques to fabricate an overall shape of parabolic reflectors. Nevertheless, large reflectors (see Fig. 1) can be composed of a set of segmented panels, which are supported by three or more adjustors on the backup structure(BUS). During the period of reflector assembly, panels need to be accurately located in the desired position with proper precision. Moreover, the surface panels will be adjusted repeatedly for a long time in order to obtain better electrical performance.

There are many methods to improve surface accuracy. A traditional method for panel adjustment is to measure the targets on the panel using theodolite and tape technique [7]. This kind of method takes much time with lower precision. 


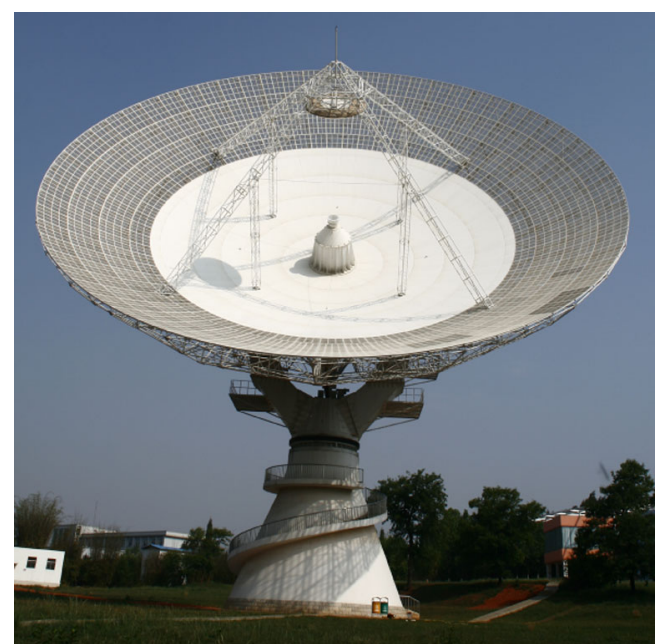

Fig. $1 \mathrm{~S} / \mathrm{X}$ dual band reflector antenna with $40 \mathrm{~m}$ diameter, TT\&C station located in Kunming for lunar exploration program

Nowadays, many industrial measuring systems such as laser ranger are broadly used in panel adjustment $[8,9]$. "Radio holography" is an advanced method to measure and adjust surface panels. This method makes use of a well-known relationship in antenna theory: the far-field radiation pattern of reflector antenna is the Fourier transformation of the field distribution in the aperture plane of antenna. Note that this relationship applies to the amplitude/phase distributions, not to the power pattern. Thus, if we can measure the radiation pattern, in amplitude and phase distribution in the antenna aperture plane with an acceptable spatial resolution. BENNETT, et al [10] presented a sufficiently detailed analysis of this method to draw the attention of radio astronomers. SCOTT and RYLE [11] used the new Cambridge $5 \mathrm{~km}$ array to measure the shape of four of the eight antennas, using a celestial radio point source and the remaining antennas to provide the reference signal Simulation algorithms were developed by RAHMAT-SAMII [12] and others [13-16], adding to the practicability of the method. Using the giant water vapour maser at $22 \mathrm{GHz}$ in Orion as a source, MORRIS, et al [17] achieved a measurement accuracy of 30 microns and were set the surface of the IRAM $30 \mathrm{~m}$ millimeter telescope to an accuracy of better than 100 microns. NIKOLIC, et al [18] described phase-retrieval holography measurements of the 100-m diameter Green Bank Telescope using astronomical sources and produced low-resolution maps of the wavefront errors. YU [19] used radio holography to correct the surface profile of the Sheshan 25-m radio telescope. Moreover, SUBRAHMANYAN [20] presented the photogrammetry in antenna metrology and proposed that the complete antenna optics have been jointly surveyed at different elevation settings for the antenna with gravity deformation.

MARTINEZ-LORENZO, et al [21] investigated near field data and reconstructed the deformed reflector surface using least square method. TANAKA, et al [22] estimated the surface error of a space reflector antenna, and corrected its influence based on antenna gain analyses. HOERNER [23] calculated panel adjustment of four-cornered panels with least square method considering internal twist in surface plate of reflector antenna. In order to keep the same wave front, DAI, et al [24] derived a method to calculate the adjustment for segmented panels of space reflector. ZHOU, et al [25] developed a new technique of contour adjustment for high precision sandwich reflector panels. The core technologies are proposed to obtain the adjustors' position and number.

In this research, a new method based on far field pattern of reflector antenna is proposed to improve the efficiency and accuracy of surface adjustment. In Sect. 1, the linear relationship between surface facet displacement in panel and electric field in far region is derived by using the method of PO. Then in Sect. 3, panel adjustment values are described as function of electric field variation with series of linear equations. The results are obtained by solving the equations with SVD. Consequently, the panel adjustment simulations and experiment results analysis are presented in Sect. 4, followed by discussions and conclusions.

\section{Computation of Far Field with PO}

$\mathrm{PO}$ is essentially an approximation that relates the surface current on a conductor with the incident electromagnetic field [26]. The induced surface current is

$\boldsymbol{J}=2\left(\hat{\boldsymbol{n}} \times \boldsymbol{H}_{\text {inc }}\right)$,

where $\hat{\boldsymbol{n}}$ is unit normal and $\boldsymbol{H}_{\text {inc }}$ is incident field. Equation (1) can be inserted into the radiation integral to find the scattered field.

According the method of $\mathrm{PO}$, the radiation integral over the reflector surface shown in Fig. 2, $\Omega$, can be expressed as

$\boldsymbol{E}(\boldsymbol{r})=-\frac{1}{4 \pi} \int_{\Omega}\left(\mathrm{j} k+\frac{1}{R}\right) \hat{\boldsymbol{R}} \times \boldsymbol{J}\left(\boldsymbol{r}^{\prime}\right) \frac{\exp (-\mathrm{j} k R)}{R} \mathrm{~d} S^{\prime}$,

where $\boldsymbol{r}$-Field point, $\boldsymbol{r}$-Source point,

$R=\left|\boldsymbol{r}-\boldsymbol{r}^{\prime}\right|$,

$\hat{\boldsymbol{R}}=\left(\boldsymbol{r}-\boldsymbol{r}^{\prime}\right) / R$,

$J\left(r^{\prime}\right)$-Surface current, $\lambda$-Wavelength, $k=2 \pi / \lambda$.

The reflector surface, $\Omega$, is meshed into a set of triangular facets $\left\{\Delta_{i}\right\}$. Since the triangles are very small is size, it is expected that $\hat{\boldsymbol{R}}$ and $R$ do not vary appreciably over a given facet. Then, replacing $\Omega$ by the triangular facets we can obtained the field as 


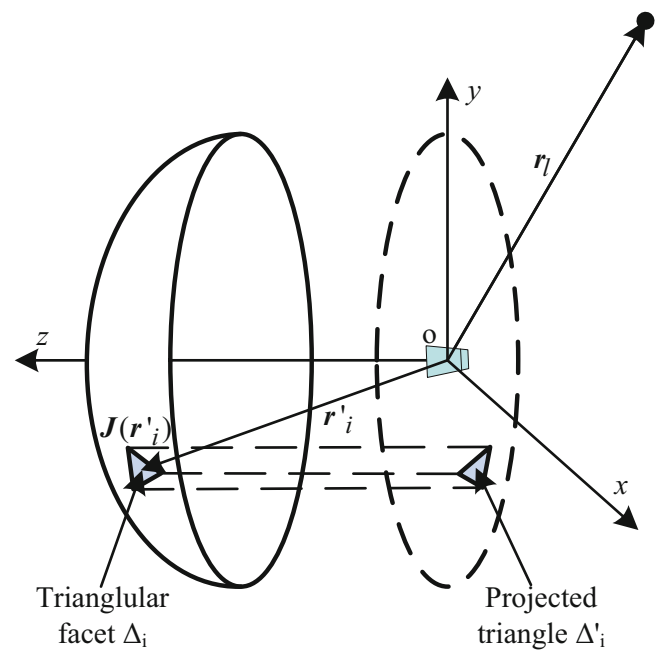

Fig. 2 Method of PO

$\boldsymbol{E}(\boldsymbol{r})=-\frac{1}{4 \pi} \sum_{i=1}^{N}\left(\mathrm{j} k+\frac{1}{R_{i}}\right) \hat{\boldsymbol{R}}_{i} \times \boldsymbol{T}_{i}(\boldsymbol{r})$.

Generally, the antenna discussed here is prime focus and the incident field can be described by

$\boldsymbol{H}_{\mathrm{s}}=\boldsymbol{h}_{\mathrm{s}}\left(\boldsymbol{r}_{i}\right) \exp \left(-\mathrm{j} k r_{s}\right) /\left(4 \pi r_{\mathrm{s} i}\right)$,

where $r_{\mathrm{s}}$-Distance to the source point, $r_{\mathrm{s} i}$-Distance from the triangle centroid to the source point, $\boldsymbol{J}_{i}\left(\boldsymbol{r}^{\prime}\right)=2 \hat{\boldsymbol{n}}_{i} \times \boldsymbol{H}_{\mathrm{s}}\left(\boldsymbol{r}^{\prime}\right)$-Equivalent surface current distribution evaluated on the $i$ th triangular facets, then

$\boldsymbol{T}_{i}(\boldsymbol{r})=\frac{\hat{\boldsymbol{n}}_{i} \times \boldsymbol{h}_{\mathrm{s}}\left(\boldsymbol{r}_{i}\right)}{2 \pi R_{i} r_{\mathrm{s} i}} \int_{\Delta i} \exp \left(-\mathrm{j} k\left(R+r_{s}\right)\right) \mathrm{d} S^{\prime}$,

$\boldsymbol{T}_{i}(\boldsymbol{r})$ can be obtained by Fourier transform of the $i$ th projected triangle $\Delta_{i}{ }^{\prime}$. Thus, the full radiation integral (2) can be expressed with the sum of all transforms of individual triangular facets.

Finally, take it for granted that the amount of panels is $N$ and there are $N_{n}$ triangle facets on the $n$th panel. In theoretical condition, the contribution of the $i$ th triangle facet to the $l$ th far field point $r_{l}$ can be described as $E_{l, n i}^{\mathrm{s}}$. The theoretical radiation field at view point $r_{l}$ contributed by the whole reflector surface can be obtained as

$\boldsymbol{E}_{l}^{\mathrm{s}}=\sum_{n=1}^{N} \boldsymbol{E}_{l, n}^{\mathrm{s}}=\sum_{n=1}^{N} \sum_{i=1}^{N_{n}} \boldsymbol{E}_{l, n i}^{\mathrm{s}}$

\section{Determination of Adjustment Value}

\subsection{Radiation Field with Facet Displacement}

Facet displacement leads to the variety of induced current, and the radiation field will vary correspondingly. Since the

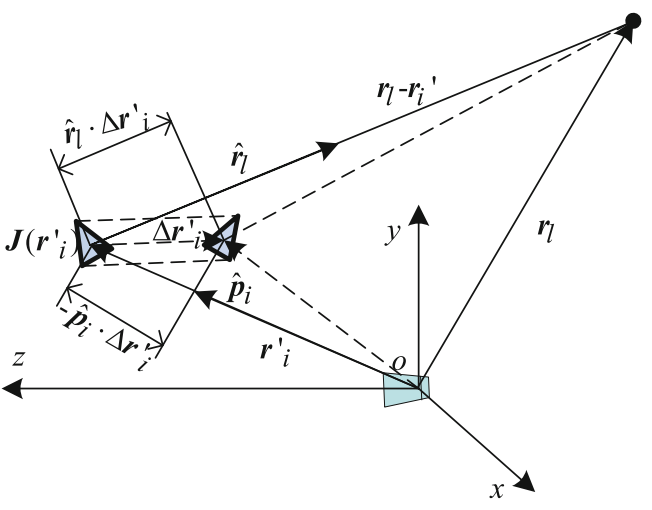

Fig. 3 Facet displacement diagram

facet displacement is very small, the induced current varies only on phase but not on amplitude [27]. If the facet offset vector is assumed as $\Delta \boldsymbol{r}_{i}^{\prime}$ shown in Fig. 3, the alterant current can be obtained as

$\boldsymbol{J}^{\Delta}\left(\boldsymbol{r}_{i}^{\prime}+\Delta \boldsymbol{r}_{i}^{\prime}\right)=\boldsymbol{J}\left(\boldsymbol{r}_{i}^{\prime}\right) \exp \left(-\mathrm{j} k \hat{\boldsymbol{p}}_{i} \cdot \Delta \boldsymbol{r}_{i}^{\prime}\right)$,

where $\hat{\boldsymbol{p}}_{i}$-Unit Poynting vector.

Put Eq. (6) into Eq. (5), the variation of radiation field in point $\boldsymbol{r}_{l}$ contributed by the $i$ th triangle facet because of the $n$th facet displacement can be expressed as

$\boldsymbol{E}_{l, n i}^{\Delta}=\boldsymbol{E}_{l, n i}^{\mathrm{s}} \exp \left(\mathrm{j} \delta_{n i}\right)$,

$\delta_{n i}=k\left(\hat{\boldsymbol{r}}_{l}-\hat{\boldsymbol{p}}_{i}\right) \cdot \Delta \boldsymbol{r}_{i}^{\prime}$.

If all facets displaced, as a result, the total scattered field in point $\boldsymbol{r}_{l}$ can be expressed as the sum of every single contribution by

$\boldsymbol{E}_{l}^{\Delta}=\sum_{n=1}^{N} E_{l, n}^{\Delta}=\sum_{n=1}^{N} \sum_{i=1}^{N_{n}} E_{l, n i}^{\Delta}$.

From Eq. (7) and Eq. (8), the variety of radiation field only by phase error is proportional to facet offset. If the phase error in Eq. (7) is small enough, the exponent function can be approximated by Taylor expansion. By taking the first two items, the variety of radiation field with phase error can be linearized as

$\boldsymbol{E}_{l, n i}^{\Delta}=\boldsymbol{E}_{l, n i}^{\mathrm{s}}\left(1+\mathrm{j} \delta_{n i}\right)$.

Based on Eq. (10), if all triangle facets displaced in the whole reflector, the influence of facet displacement on the radiation field forms a linear system.

\subsection{Relationship Between Adjustment and Displacement}

Because individual panels are generally fabricated much more precisely than fixed onto a large BUS, the panels can be assumed to be rigid plate. Thus, the panel position is 


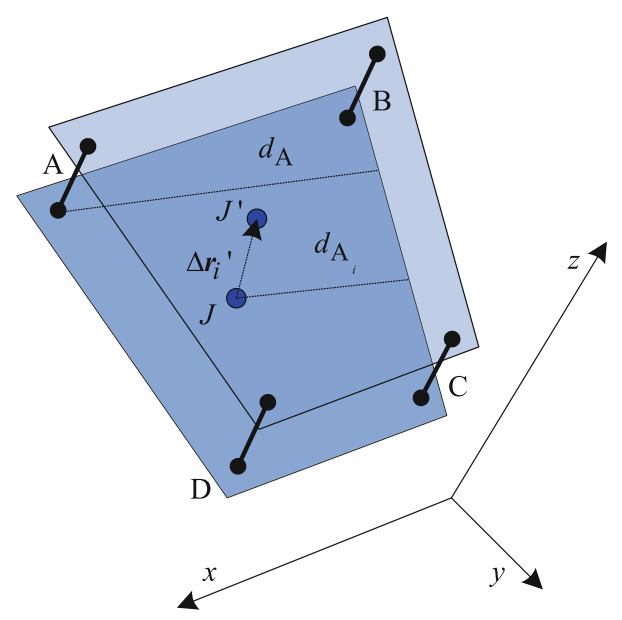

Fig. 4 Panel adjustment diagram

only geometrically decided by three adjustors selected in all corner screws in Fig. 4.

Once the panel is adjusted by three adjustors, the facet displacement in this panel can be written as $[28,29]$

$\Delta \boldsymbol{r}_{i}^{\prime}=\boldsymbol{S}_{n i} \boldsymbol{a}_{n}$,

where $\boldsymbol{a}_{n}$-Panel adjustment vector, $\boldsymbol{S}_{n i}$-Transform matrix between adjustment vector and displacement of the $i$ th facet.

$\boldsymbol{a}_{n}=\left[\begin{array}{lll}a_{\mathrm{A}} & a_{\mathrm{B}} & a_{\mathrm{C}}\end{array}\right]^{\mathrm{T}}$,

$\boldsymbol{S}_{n i}=\left[\begin{array}{lll}\operatorname{signA} & \frac{d_{\mathrm{A}_{i}}}{d_{\mathrm{A}}} \overline{\boldsymbol{n}}_{\mathrm{A}_{\mathrm{i}}} & \operatorname{signB}_{i} \frac{d_{\mathrm{B}_{i}}}{d_{\mathrm{B}}} \overline{\boldsymbol{n}}_{\mathrm{B}_{\mathrm{i}}} \quad \operatorname{signC}_{i} \frac{d_{\mathrm{C}_{i}}}{d_{\mathrm{C}}} \overline{\boldsymbol{n}}_{\mathrm{C}_{\mathrm{i}}}\end{array}\right]^{\mathrm{T}}$

where $\overline{\boldsymbol{n}}$-Unit normal vector on the facet.

$\overline{\boldsymbol{n}}=\left[\begin{array}{lll}n_{x} & n_{y} & n_{z}\end{array}\right]^{\mathrm{T}}$

Actually, reflector panels are designed for curve fitting to a parabolic surface. As a result, facet displacement includes components in three different directions when different adjustors adjusted. Therefore, the whole offset vector is the superposition of three components.

\subsection{Modeling of Linear System}

The total radiation field of point $r_{l}$ can be expressed as the overall contribution of the $n$th panel because of its facet displacement, say, putting Eq. (10) and Eq. (11) into Eq. (9) as

$\boldsymbol{E}_{l, n}^{\Delta}=\boldsymbol{E}_{l, n}^{\mathrm{s}}+\boldsymbol{B}_{n} \boldsymbol{a}_{n}$,

$\boldsymbol{B}_{n}=\sum_{i=1}^{N_{n}}\left[\boldsymbol{E}_{l, n i}^{\mathrm{s}} \mathrm{j} k\left(\hat{\boldsymbol{r}}_{l}-\hat{\boldsymbol{p}}_{n i}\right) \boldsymbol{S}_{n i}\right]$.

The total radiation field of all $m$ observing points in the far region can be obtained by adding the contributions of all panels together. Noting that

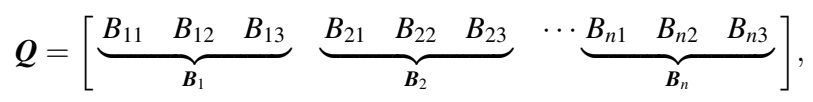

$\boldsymbol{M}=\left[\begin{array}{llll}\boldsymbol{Q}_{1} & \boldsymbol{Q}_{2} & \cdots & \boldsymbol{Q}_{m}\end{array}\right]^{\mathrm{T}}$,

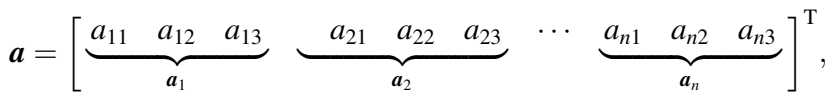

thus, the relationship between the adjustment values of all panels and the electric field in far region can be written into a linear system as

$\boldsymbol{E}^{\Delta}=\boldsymbol{E}^{\mathrm{S}}+\boldsymbol{M a}$,

\subsection{Solution of Linear Equations Using SVD}

The method of Singular Value Decomposition (SVD) is used to calculate the pseudo inverse matrix $\boldsymbol{B}$. In SVD the $m \times n$ matrix $\boldsymbol{A}$ (where $m \geq n$ )can be expressed as the product of three matrices:

$\boldsymbol{A}=\boldsymbol{U} \boldsymbol{W} \boldsymbol{V}^{\mathrm{T}}$,

where $\boldsymbol{U}$-An $m \times n$ column orthogonal matrix, $\boldsymbol{W}-\mathrm{An}$ $n \times n$ diagonal matrix whose elements $w_{i}$ are positive or zero and are referred to as the singular values of the matrix $\boldsymbol{A}, \boldsymbol{V}$-An $n \times n$ orthogonal matrix,T-Transpose operator.

The matrix $\boldsymbol{B}$ can be obtained as

$\boldsymbol{B}=\boldsymbol{V} \boldsymbol{W}^{-1} \boldsymbol{U}^{\mathrm{T}}$,

where $1 / 1 w_{j}$ in $\boldsymbol{W}^{-1}$ can be replaced by 0 in the event that $w_{j}=0$. The matrix $\boldsymbol{V}$ defines a basic set of modes which are essentially unique orthonormal, so that any arbitrary configuration of the system can be written as a unique linear combination of these basic modes [30]. Using SVD to solve Eq. (23), the generalized reverse of transformation matrix can be calculated. After that, the panel adjustment values on the whole reflector can be obtained from the following equations

$\boldsymbol{M a}=\boldsymbol{E}^{\Delta}-\boldsymbol{E}^{\mathrm{S}}$.

\section{Experiment and Analysis}

The effect of facet displacement on radiation field is demonstrated on a $3.7 \mathrm{~m}$ diameter reflector antenna, which has 12 panels. Each panel is fixed on the BUS using 4 screws. 3 of them are chosen as adjustors for panel adjustment. Two panels in the right zone of the reflector shown in Fig. 5 are displaced by filling gaskets with $3 \mathrm{~mm}$ thickness between panels and adjustors (see Fig. 6) to simulate panel alignment error. 


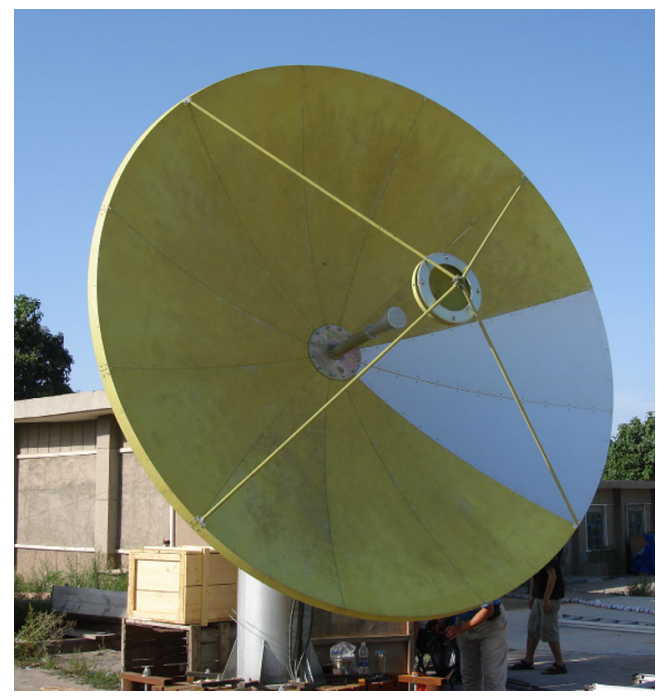

Fig. 5 Panel distribution in reflector surface (front view)

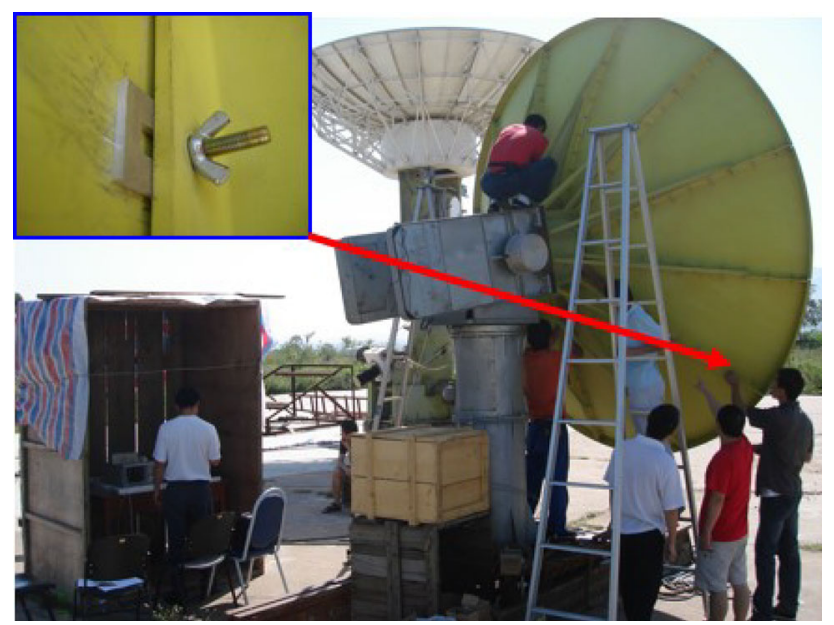

Fig. 6 Panel displacement by filling gaskets (back view)

The focus/diameter ratio is 0.35 and the subreflector diameter is $0.44 \mathrm{~mm}$. The far field of the antenna is tested at $12.5 \mathrm{GHz}$, illuminated by a taper ripple horn. The electric field in aperture plane can be assumed as

$E_{0}\left(\overline{\rho^{\prime}}\right)= \begin{cases}1-0.9 \exp \left(\overline{\rho^{\prime}}-1\right), & \overline{\rho^{\prime}} \geq 0.5, \\ 1-0.85 \exp \left(0.13-\overline{\rho^{\prime}}\right), & \overline{\rho^{\prime}}<0.5,\end{cases}$

where $\overline{\rho^{\prime}}$-Normalized radius, which is in $[0,1]$.

\subsection{Experimental Results and Analysis}

According to $\mathrm{PO}$, the surface is meshed into lots of triangle facets. The size of each facet is less than $\lambda / 4$, therefore there are totally 262848 triangle facets all over the reflector surface. Utilizing the method mentioned above, the radiation field with and without facet displacements are

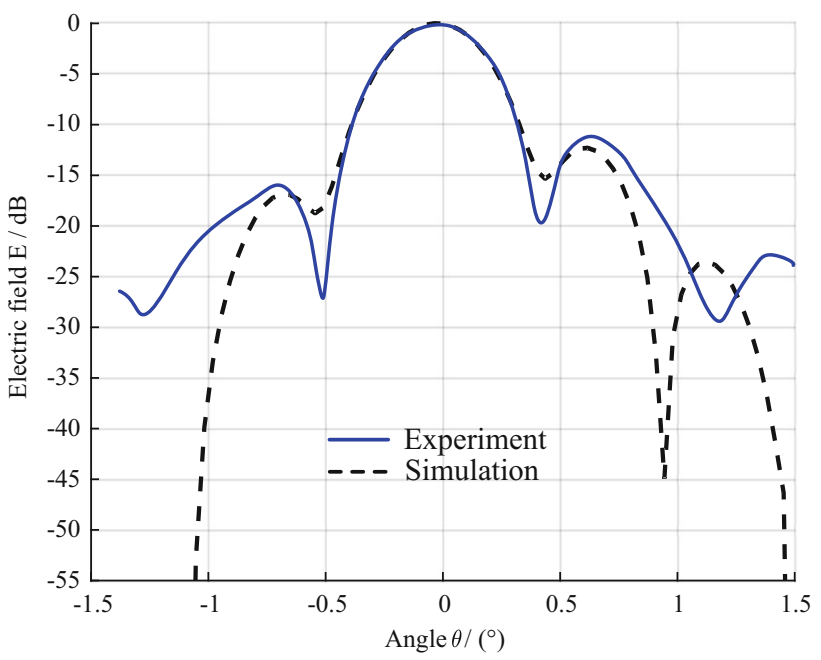

Fig. 7 Comparison of simulation with experiment

Table 1 Electrical performance comparison between simulation and experiment results

\begin{tabular}{lllllll}
\hline Performance & $\mathrm{A}_{0}$ & $\mathrm{~B}_{0}$ & Error & $\mathrm{A}_{1}$ & $\mathrm{~B}_{1}$ & Error \\
\hline Gain $(\mathrm{dB})$ & 52.84 & 52.23 & 0.61 & 52.21 & 52.00 & 0.21 \\
HPBW $\left(^{\circ}\right)$ & 0.390 & 0.380 & 0.01 & 0.48 & 0.49 & 0.01 \\
$\begin{array}{l}\text { Left SLL } \\
\quad-14.33\end{array}$ & -14.54 & 0.21 & -17.3 & -17.8 & 0.5 \\
$\begin{array}{l}\text { Right SLL } \\
\quad-14.33\end{array}$ & -14.18 & -0.15 & -13.85 & -12.53 & -1.32 \\
$\quad$ & & & & & & \\
\hline
\end{tabular}

Note: $A_{0}$ and $B_{0}$ are simulation and experiment without displacement. $A_{1}$ and $B_{1}$ are with $3 \mathrm{~mm}$ displacement. HPBW means half power beam width. SLL means side lobe level

calculated. At the same time, the electric field in far region is also tested.

After the selected panels displaced, the far field pattern is tested. The experimental data is used to compared with simulation results shown in Fig. 7 and Table 1. Both the curves fit very well, which tell us that the presented effect model is adaptable to show the influence of facet displacement on the electric field in far region.

\subsection{Simulations for Panel Adjustment}

From Eq. (23), a vector of adjustment value can be obtained. Since the transformation matrix $\boldsymbol{M}$ is generally not a full rank matrix, the solution is not exclusive in Eq. (21). The adjustment values are not in accordance with the thickness of testing gaskets, because different panel positions have probably the same corresponding far field pattern. However, in practice, a feasible solution means that the electrical performance can be improved as well as possible with panel adjustment only once. 


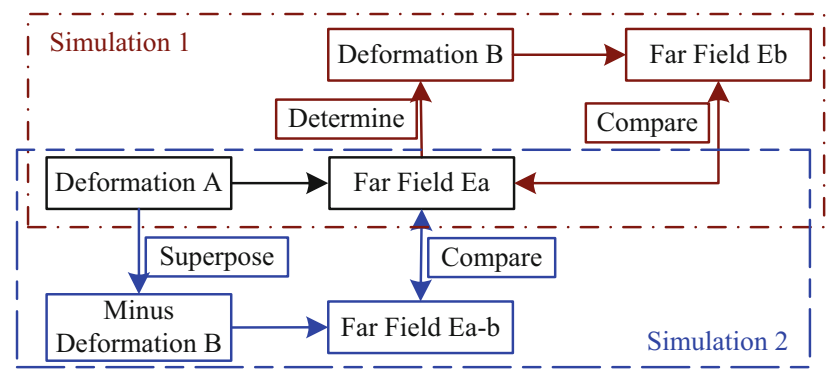

Fig. 8 Flow chart of simulations for panel adjustment

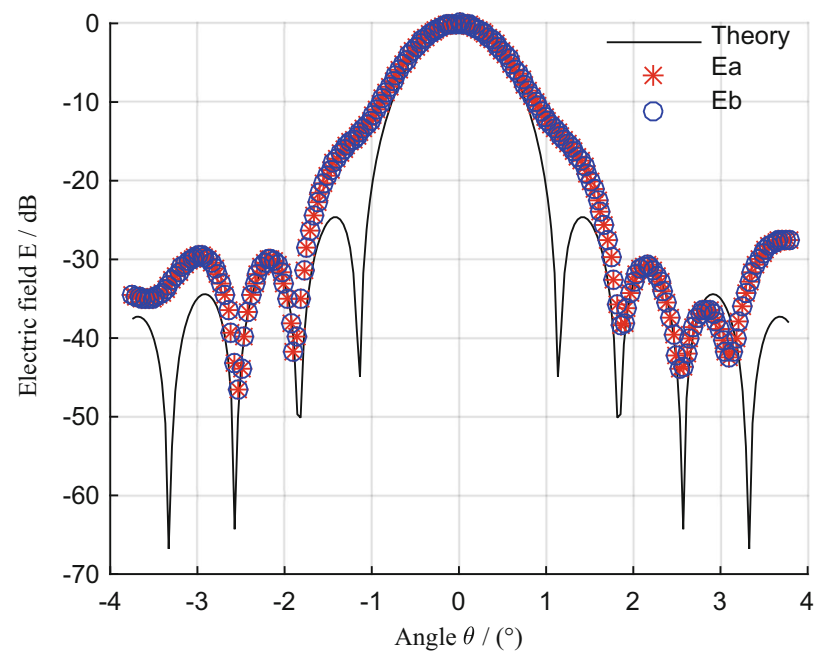

Fig. 9 Calculation results of simulation 1

Two simulations (see Fig. 8) are set to verify the efficiency of panel adjustment value calculating by Eq. (23). In simulation 1 , deformation $\mathrm{A}$ is assumed on reflector surface and the correspondent far field pattern is Ea. Then, using Eq. (23), a set of panel adjustment value can be calculated. Putting the solution as panel displacement onto the theoretical surface, which results in deformation $\mathrm{B}$ and the correspondent far field pattern Eb. From Fig. 9, Eb and Ea fit very well, which shows that the deformation B calculated by Eq. (21) and the supposed deformation A are consistent. So, the presented method can be used to determinate panel displacement from far field pattern.

As for simulation 2 in Fig. 8, supposed deformation A is superposed with minus deformation $\mathrm{B}$, and the correspondent far field patterns are Ea-b shown in Fig. 10. It demonstrates that panel adjustment value from Ea is utilized to diminish the deformation $\mathrm{A}$. At the same time, $\mathrm{Ea}-\mathrm{b}$ is much better than the initial value of far field pattern Ea. Therefore, the panel adjustment values achieved by the presented method could significantly improve the far field pattern of reflector antenna with panel displacement.

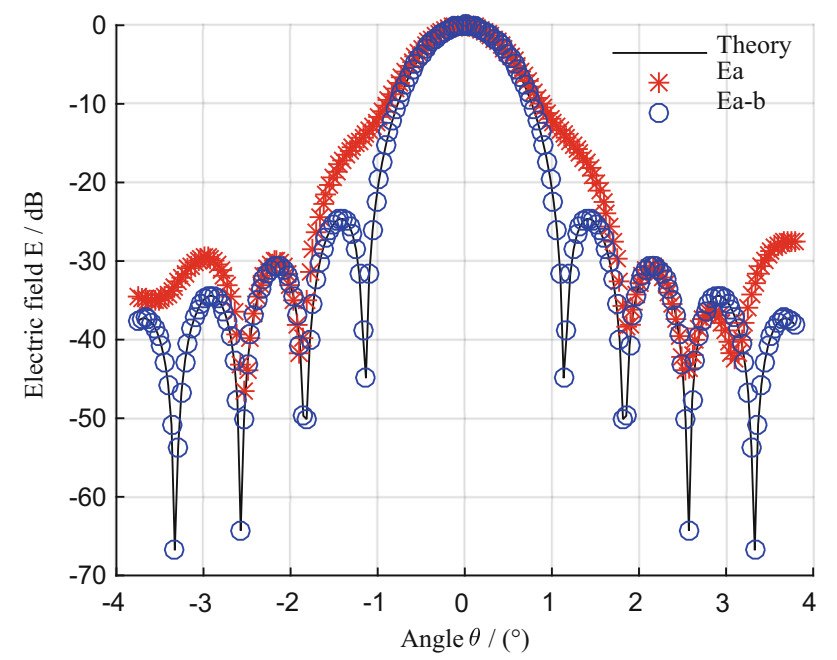

Fig. 10 Calculation results of simulation 2

Table 2 Error analysis for the linearization using Eq. (10)

\begin{tabular}{lclcl}
\hline$\delta / \mathrm{rad}$ & $\cos \delta / \%$ & $\sin \delta / \%$ & $|\boldsymbol{E}| / \%$ & $\varepsilon$ \\
\hline$-0.3167 \sim+0.3167$ & 5.23 & 1.69 & 5 & $\lambda / 40$ \\
$-0.4485 \sim+0.4485$ & 10.98 & 3.43 & 10 & $\lambda / 28$ \\
$-0.55 \sim+0.55$ & 17.3 & 5.23 & 15 & $\lambda / 23$ \\
\hline
\end{tabular}

\subsection{Discuss of the Validity Range}

According Eq. (10), the facet displacement is considered as phase error in exponential function during calculating radiation field. In order to construct the linear system of panel adjustment, the exponential item should be linearized by taking the first two items of a Tylor expansion, which results in $\exp \left(\delta_{n i}\right)=1+\mathrm{j} \delta_{n i}$. The approximation must bring some errors in calculating far field. The electric field can be divided into real and imaginary parts, shown as $\exp \left(\mathrm{j} \delta_{n i}\right)=$ $\cos \delta_{n i}+\mathrm{j} \sin \delta_{n i}$. The fitting errors are given in Table 2 .

In Table 2, the approximation of exponential function can meet the accuracy only for very small error. In order to overcome this shortcomings, a fitting method is used to handle higher order items, in which the exponential function is fitted by several piecewise lines. All the items of Taylor's expansion can be divided into two parts, real parts and imaginary parts, shown as

$$
\begin{array}{r}
\exp (\mathrm{j} \delta)=\underbrace{\left(\frac{\delta^{0}}{0 !}-\frac{\delta^{2}}{2 !}+\frac{\delta^{4}}{4 !}+\cdots\right)}_{p}+\underbrace{\left(\mathrm{j} \frac{\delta}{1 !}-\mathrm{j} \frac{\delta^{3}}{3 !}+\mathrm{j} \frac{\delta^{5}}{5 !}+\cdots\right)}_{q} \\
\approx(a+b \delta)+\mathrm{j}(c+d \delta)=(a+\mathrm{j} c)+(b+\mathrm{j} d) \delta,
\end{array}
$$


Table 3 Parameters of piecewise linear function for fitting real parts in Eq. (25) with $p=4$ and $q=4$

\begin{tabular}{llllcc}
\hline Amount of piecewise lines $p$ & Phase error $\delta /$ rad & Path length error $\varepsilon / \lambda$ & Parameter $a$ & Parameter $b$ & Fitting error $e$ \\
\hline 1 & {$[-3.14,-1.86)$} & {$[-0.250,-0.148)$} & 0.9778 & 0.7134 \\
2 & {$[-1.86,-1.58)$} & {$[-0.148,-0.126)$} & 1.5623 & 0.9955 & 0.0494 \\
3 & {$[-1.58,-1.49)$} & {$[-0.126,-0.119)$} & 1.5746 & 1.003 & 0.0119 \\
4 & {$[-1.49,-0.73)$} & {$[-0.119,-0.058)$} & 1.4139 & 0.8831 & 0.0493 \\
5 & {$[-0.73,0.37)$} & {$[-0.058,0.029)$} & 0.9654 & 0.1736 \\
6 & {$[0.37,1.32)$} & {$[0.029,0.105)$} & 1.2562 & -0.731 & 0.0494 \\
7 & {$[1.32,1.56)$} & {$[0.105,0.124)$} & 1.5588 & -0.9925 \\
8 & {$[1.56,1.68)$} & {$[0.124,0.134)$} & 1.5762 & -1.004 & 0.0125 \\
9 & {$[1.68,2.61)$} & {$[0.134,0.208)$} & 1.327 & -0.87 & 0.0406 \\
10 & {$[2.61,4.1)$} & {$[0.208,0.326)$} & 1.1673 & -0.7656 & 0.0489 \\
\hline
\end{tabular}

Table 4 Parameters of piecewise linear function for fitting Imaginary parts in Eq. (25) with $p=4$ and $q=4$

\begin{tabular}{lllccc}
\hline Amount of piecewise lines $q$ & Phase error $\delta /$ rad & Path length error $\varepsilon / \lambda$ & Parameter $c$ & Parameter $d$ & Fitting error $e$ \\
\hline 1 & {$[-3.14,-3.03)$} & {$[-0.250,-0.241)$} & -3.6463 & -1.1844 & 0.045 \\
2 & {$[-3.03,-2.49)$} & {$[-0.241,-0.198)$} & -3.1153 & -1.0051 & 0.0496 \\
3 & {$[-2.49,-1.41)$} & {$[-0.198,-0.112)$} & -1.6064 & -0.3714 & 0.0499 \\
4 & {$[-1.41,-0.39)$} & {$[-0.112,-0.031)$} & -0.2044 & 0.6052 & 0.0487 \\
5 & {$[-0.39,-0.01)$} & {$[-0.031,-0.001)$} & -0.0021 & 0.9764 & 0.035 \\
6 & {$[-0.01,0.01)$} & {$[-0.001,0.001)$} & 0 & 0 & 0.9701 \\
7 & {$[0.01,0.44)$} & {$[0.001,0.035)$} & 0.003 & 0.5578 & 0.0492 \\
8 & {$[0.44,1.48)$} & {$[0.035,0.118)$} & 0.2466 & -0.4177 & 0.0489 \\
9 & {$[1.48,2.54)$} & {$[0.118,0.202)$} & 1.7001 & -1.0173 & 0.0429 \\
10 & {$[2.54,3.04)$} & {$[0.202,0.242)$} & 3.1502 & -1.2213 & 0.0482 \\
\hline
\end{tabular}

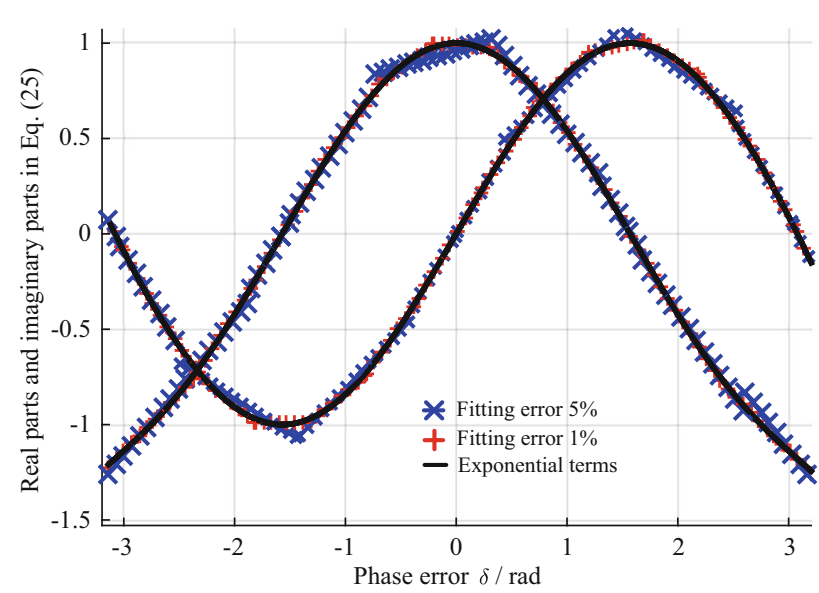

Fig. 11 Fitting exponential function using piecewise lines

where $p$-Amount of real parts, $q$-Amount of imaginary parts.

By changing $p$ and $q$, the parameters of piecewise linear function can be obtained. When $p=4$ and $q=4$, the fitting error is less than 5\%, shown in Table 3 and Table 4. For the whole domain of $[-3.14,3.14]$, the method of

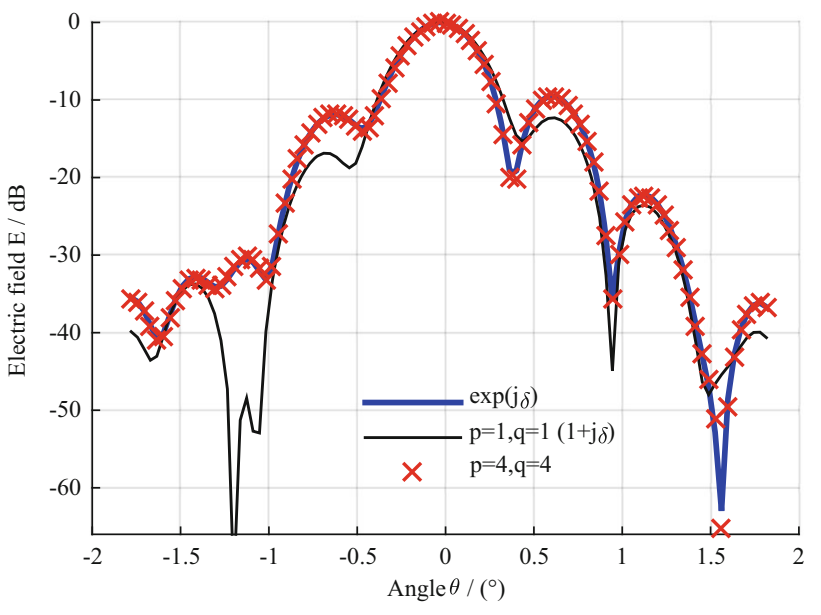

Fig. 12 Far field simulation with different $p$ and $q$

piecewise lines can also satisfy the fitting accuracy with $p=10$ and $q=11$.

If the fitting accuracy increases form $5 \%$ to $1 \%$, the fitting of piecewise lines is very strict for both real and imaginary parts, with $p=15$ and $q=20$, shown in Fig. 11. 
The electric field in far regions are calculated with different $p$ and different $q$, shown in Fig. 12. From the comparison, the far field is more sensitive to $p$ than to $q$. Equation (10) is the special circumstances with $p=1$ and $q=1$. It can be concluded from Fig. 12 that the piecewise linear fitting method is suitable to substitute the exponential function about phase error with $p=4$ and $q=4$. Nevertheless, the method of Eq. (23) can utilize this kind of approximation, just only multiplying the proper parameter to each element in transformation matrix $\boldsymbol{M}$.

\section{Conclusions}

(1) According to the method of PO, the effect model of facet displacement on the electric field in far region of reflector antenna is developed. Thus, the equation of integral for calculating far field pattern of antenna with distorted surface is transformed into a summation of many displaced facets.

(2) A linear system is constructed for calculating panel adjustment values. And then SVD is used to solve the linear equations. As a result, all adjustment values of panel adjustors are obtained. Therefore, the accuracy of reflector surface is improved significantly because the actual target of panel adjustment is to make the electrical performance better.

(3) A reflector antenna with $3.7 \mathrm{~m}$ diameter and 12 panels is taken to verify the effect model between facet displacement and radiation field. With sets of experiments, the method shows a good performance, and a good agreement is found between the simulation curves and experimental curves as well. The results verify the efficiency of presented method which has such an advantage that all panels over the reflector surface are adjusted as a whole.

(4) For larger phase error, the exponential function can be approximated with 4 piecewise lines and the fitting error is less than $5 \%$. The validation range of the method is expanded by using piecewise lines to fit the exponential function.

Open Access This article is distributed under the terms of the Creative Commons Attribution 4.0 International License (http://creative commons.org/licenses/by/4.0/), which permits unrestricted use, distribution, and reproduction in any medium, provided you give appropriate credit to the original author(s) and the source, provide a link to the Creative Commons license, and indicate if changes were made.

\section{References}

1. RAHMAT-SAMII Y, DENSMORE A. Reflector antenna developments: a perspective on the past, present, and future[J]. IEEE Antennas and Propagation Magazine, 2015, 57(2): 85-95.
2. WIJNHOLDS S J, TOL S V D, NIJBOER R, et al. Calibration challenges for future radio telescopes[J]. IEEE Signal Processing Magazine, 2010, 27(1): 30-42.

3. USOFF J M, CLARKE M T, LIU Chao, et al. Optimizing the HUSIR antenna surface[J]. Lincoln Laboratory Journal, 2014, 21(1): 83-105

4. LENG Guojun, WANG Wei, DUAN Baoyan, et al. Field-coupling model of reflector antenna with electromagnetic and displacement fields and its application to reflector antenna with the diameter of $65 \mathrm{~m}[\mathrm{~J}]$. Chinese Journal of Mechanical Engineering, 2012, 48(23): 1-9. (in Chinese)

5. WANG Meng, WANG Wei, WANG Congsi, et al. A practical approach to evaluate the effects of machining errors on the electrical performance of reflector antennas based on paneled forms[J]. IEEE Antennas Wireless and Propagation Letters, 2014, 13: 1341-1344.

6. LIAN Peiyuan, DUAN Baoyan, WANG Wei, et al. A mesh refinement method of reflector antennas using quadratic surface construction over each structure element[J]. IEEE Antennas Wireless and Propagation Letters, 2014, 13: 1557-1560.

7. KESTEVEN M J, PARSONS B F, YABSLEY D E. Antenna Reflector metrology: the australia telescope experience[J]. IEEE Transaction on Antennas and Propagation, 1988, 36(10): 1481-1484

8. IMBRIALE W A, BRITCLIFFE M J, BRENNER M. Gravity deformation measurement of NASA's deep space network 70meter reflector antennas[R]. JPN Progress Report, 2001: 42-127.

9. WANG Jinjiang, YANG Zhiwen. A Surface measurement system from China for large antennas[J]. IEEE Instrumentation \& Measurement Magazine, 2002, 5(1): 20-22.

10. BENNETT J C, ANDERSON A P, MCINNES $\mathrm{P}$ A, et al. Microwave holographic metrology of large reflector antennas[J]. IEEE Transaction on Antennas and Propagation, 1976, 24(3): 295-303.

11. SCOTT P F, RYLE M. A Rapid Method for measuring the figure of a radio telescope reflector[J]. Monthly Notices Roy. Astron. Soc., 1977, 178: 539-545.

12. RAHMAT-SAMII Y. Microwave holography of large reflector antennas - simulation algorithms[J]. IEEE Transaction on Antennas and Propagation, 1985, 33(11): 1194-1203.

13. GODWIN M P, SCHOESSOW E P, GRAHL B H. Improvement of the Effelsberg 100 meter telescope based on holographic reflector surface measurement[J]. Astronomy and Astrophysics, 1986, 167: 390-394.

14. ROCHBLATT D J, SEIDEL B L. Microwave antenna holography[J]. IEEE Transaction on Microwave Theory and Techniques, 1992, 40(6): 1 249-1 300.

15. JAMES G C, POULTON G T, MCCULLOCH P M. Panel setting from microwave holography by the method of successive projections[J]. IEEE Transaction on Antennas and Propagation, 1993, 41(11): 1523-1529.

16. LEGG T H, AVERY L W, MATTHEWS H E, et al. Gravitational deformation of a reflector antenna measurement with single-receiver holography[J]. IEEE Transaction on Antennas and Propagation, 2004, 52(1): 20-25.

17. MORRIS D, BAARS J W M, HEIN H, et al. Radio-holographic reflector measurement of the $30-\mathrm{m}$ millimeter radio telescope at $22 \mathrm{GHz}$ with a cosmic signal source[J]. Astronomy and Astrophysics, 1998, 203: 399-406.

18. NIKOLIC B, PRESTAGE R M, BALSER D S, et al. Out-offocus Holography at the green bank telescope[J]. Astronomy and Astrophysics, 2007, 465: 685-693.

19. YU Hong. Microwave holography measurement and adjustment of $25 \mathrm{~m}$ radio telescope of Shanghai[J]. Microwave and Optical Technology Letters, 2007, 49(2): 467-470. 
20. SUBRAHMANYAN R. Photogrammetric measurement of the gravity deformation in a Cassegrain antenna[J]. IEEE Transaction on Antennas and Propagation, 2005, 53(8): 2590-2596.

21. MARTINEZ-LORENZO J A, GONZALEZ-VALDES B, RAPPAPORT C, et al. Reconstructing distortions on reflector antennas with the iterative-field-matrix method using near-field observation data[J], IEEE Transactions on Antennas and Propagation, 2011, 59(6): 2434-2437.

22. TANAKA H. Surface error estimation and correction of a space antenna based on antenna gain analyses[J]. Acta Astronautica, 2011, 68: 1062-1069.

23. HOERNER S. Internal twist and least-squares adjustment of four-cornered surface plate for reflector antennas[J]. IEEE Transactions on Antennas and Propagation, 1981, 29(6): 953-958.

24. DAI Yanfeng, LIU Zaozhen. Research on cophasing optimized algorithms for space-based telescope segmented primary mirror[J]. Optical Technique, 2008, 34(3): 914-916. (in Chinese)

25. ZHOU Xianbin, HAO Changling, LI Dongsheng, et al. New technique of contour adjustment for high precision reflector panels[J]. Journal of Beijing University of Aeronautics and Astronautics, 2009, 35(2): 127-131. (in Chinese)

26. IMBRIALE W A. Large antennas of the deep space network[M]. 1st ed. New York: John Wiley \& Sons, Inc., 2003.

27. MARTINEZ-LORENZO J A, PINO A G, VEGA I, et al. ICARA: Induced-current analysis of reflector antennas[J]. IEEE Antenna and Propagation Magazine, 2005, 47(2): 92-100.

28. YI Wei, JIANG Zhaoliang, SHAO Weixian, et al. Error Compensation of Thin Plate-shape Part with Prebending Method in Face Milling[J]. Chinese Journal of Mechanical Engineering, 2015, 28(1): 88-95.

29. WANG Wei, DUAN Baoyan, LI Peng, et al. Optimal surface adjustment by the error transformation matrix for a segmented reflector antenna[J]. IEEE Antennas and Propagation Magazine, 2010, 52(3): 80-88.
30. CHANAN G, MACMARTIN D G, NELSON J, et al. Control and alignment of segmented-mirror telescopes: matrices, modes, and error propagation[J]. Applied Optics, 2004, 43(6): 1223-1232.

Wei WANG, born in 1980, is currently an associate professor at Key Laboratory of Electronic Equipment Structure Design of Ministry of Education, Xidian University, China. His main research interests include electro-mechanical coupling theory and multidisciplinary optimization in reflector antennas and phased arrays. Tel: +86-2988203040; E-mail: wwang@xidian.edu.cn

Peiyuan LIAN, born in 1989 , is currently a $\mathrm{PhD}$ candidate at Key Laboratory of Electronic Equipment Structure Design of Ministry of Education, Xidian University, China. His research interests include integrated structural electromagnetic design of reflector antennas. E-mail: lian100fen@126.com

Shuxin ZHANG, born in 1987, is currently a lecturer at Key Laboratory of Electronic Equipment Structure Design of Ministry of Education, Xidian University, China. His research interests include integrated structural electromagnetic optimization design of cable mesh antennas and structural optimization. E-mail: sxzhang@xidian.edu.cn

Binbin XIANG, born in 1984, is currently a $\mathrm{PhD}$ candidate at Xidian University and an Engineering at the Xinjiang Astronomical Observation, Chinese Academy of Sciences, China. His research interests include shape design and adjustment of reflector panels for large radio telescopes. E-mail: xiangbinbin@xao.ac.cn

Qian XU, born in 1981, is currently an Engineering at the Xinjiang Astronomical Observation, Chinese Academy of Sciences, China. His research interests include structural design of radio telescope. E-mail: xuqian@xao.ac.cn 